\title{
La Alfabetización inicial en Venezuela. Desde la Escuela Nueva hasta las Escuelas Bolivarianas
}

\author{
Initial Literacy in Venezuela. From the New School to the Bolivarian Schools.
}

\begin{abstract}
Autora
Joamsner Magdalena Arévalo. Universidad Nacional Experimental Francisco de Miranda.

Falcón.Venezuela.smartkelly1912@gmail.com https://orcid.org/0000-0001-7617-7265

Fecha de recibido: 2019-07-05

Fecha de aceptado para publicación: 2019-08-20

Fecha de publicación: 2019-09-30

\section{Resumen}

Actualmente los ciudadanos están expuestos a una gran cantidad de información presentada a través de diversos medios, lo que exige destrezas que no se limiten a la reproducción y decodificación sino que los mismos deben ser capaces de interpretar y expresarse a través de distintos medios y en más de un idioma. En este sentido los conceptos relacionados con las prácticas alfabetizadoras alrededor del mundo se han ido transformando hacia un enfoque más comunicativo en el que la producción y comprensión del discurso escrito se aprecie como una herramienta de crecimiento personal incluyendo el uso de las Tecnologías de la Información Comunicación (TIC). Con esto en mente se expone una investigación de corte documental con el objetivo de describir el recorrido histórico del concepto de alfabetización en el Sistema Educativo Venezolano (SEV), comenzando en 1936 con la Escuela Nueva hasta el actual Sistema Educativo Bolivariano (SEB), esto permite apreciar la transformación del concepto y las prácticas alfabetizadoras en el país. Este estudio aporta como conclusión principal que no solo no hay un consenso sobre la visión y metodología para la alfabetización en Venezuela, sino que se hace necesario un replanteamiento de dicho concepto.
\end{abstract}

Palabras clave: Alfabetización; desaprendizaje; racionalismo crítico; recorrido histórico.

\begin{abstract}
Currently citizens are exposed to a large amount of information presented through various means. This torrent of information requires skills that are not only limited to the reproduction and decoding of signs and symbols but, individuals must be able to interpret and express themselves through different means and in more than one language. In this sense, the concepts related to literacy practices around the world have been transformed towards a more communicative approach in which the production and understanding of written discourse is appreciated as a tool for personal growth, including in this the use of Information and Communication Technology (ICT). With this in mind, a documentary research is exposed with the objective of describing a historical journey of the transformation of the concept of literacy in the Venezuelan Educational System (VES), beginning on 1936 with the new school until the current Bolivarian Educational System (BES). This will allow to appreciate that not only is there no consensus on the vision and methodology for literacy in Venezuela, but also a rethinking of the concept becomes necessary.
\end{abstract}

Keywords: Literacy; un-learning; critical rationalism; historical journey. 


\section{Introducción.}

\section{Alfabetizar. Una reflexión sobre el concepto}

La comunicación, específicamente la escritura es signo inequívoco de la condición humana. El lenguaje es un fenómeno dinámico revestido de creatividad, productividad y complejidad, con respecto a eso Ferreiro (2016) afirma que son las reglas y parámetros de articulación los que definen un lenguaje, pues las palabras por sí solas no representan nada hasta que son combinadas para formar oraciones que construyan un mensaje inteligible.

Al proceso de aprendizaje del lenguaje escrito se le denomina alfabetización y se define como el acto de aprendizaje, creación e interpretación del lenguaje escrito con el propósito de comunicarse. En este sentido, escribir o leer son formas de usar el lenguaje para accionar y conseguir objetivos. Leemos y escribimos con el fin de persuadir, argumentar, solicitar, regular comportamientos, entre otras cosas (Portolés, 2016).

Tal como lo exponen Hernándo y García (2016) y Cassany (2015) la escritura es y sigue siendo el código más usado para la interacción humana, incluso en comunidades virtuales. Continuando con los mismos autores, la comunicación vía internet ha promovido el acercamiento a la información desde el punto de vista de la competencia multimodal, en la cual el individuo representa y aborda los mensajes desde una variedad de sistemas: habla, escritura, imagen, audio de modo que lo que comenzó como un documento escrito se convierte en una comunidad de expresión. De allí que, ya no se puede limitar la alfabetización al manejo de los signos y símbolos delimitándolo al uso del lápiz y papel, sino que se debe abarcar el uso y manejo de los medios audiovisuales para crear, representar, compartir, comprender e interpretar información en diversos medios de interacción humana y con distintos propósitos.

Con respecto a esto, la UNESCO (2006, p. 13) se refiere a la alfabetización como: la "habilidad para identificar, entender, interpretar, crear, comunicar, y calcular mediante el uso de materiales escritos e impresos relacionados con distintos contextos". Owens (2016) a su vez, define el término alfabetización como la capacidad de comprender y manejar distintos dominios culturales y tecnológicos. Cada día el término abarca más y más habilidades, por ejemplo, el uso eficiente de los computadores, teléfonos inteligentes, conocimiento básico de más de un idioma, así como de terminología y modismos globales. 
Desde esta perspectiva una persona alfabetizada sería aquella que es capaz de comprender, producir y básicamente interactuar con todos los elementos y en todas las situaciones dentro de la sociedad a la que pertenece. No se trata, entonces, de saber leer y escribir, se trata de ser capaz de interpretar y manejar la información que produce la comunidad global. Ser estratégicamente competente en todos los contextos actuales de comunicación (Padrón, 2013).

Se procede entonces a una investigación documental con el objetivo de describir el recorrido histórico del concepto de alfabetización en el Sistema Educativo Venezolano, comenzando en 1936 con la Escuela Nueva hasta el actual Sistema Educativo Bolivariano, lo que permitirá apreciar la transformación del concepto y las prácticas alfabetizadoras en el país. La importancia de esta investigación se perfila en que es el concepto lo que marcará la pauta de las políticas, presupuesto y todas las acciones educativas de un país (Braslavsky, 2003).

\section{Metodología}

Este trabajo se origina a partir de una investigación documental de las fuentes de información oficial disponibles en relación a las prácticas de alfabetización dentro del Sistema Educativo Venezolano. No está de más destacar que la investigación documental parte de la recolección, organización, análisis e interpretación de información proveniente de fuentes bibliográficas sean estas físicas o electrónicas (Bernal, Martínez, Parra y Jiménez, 2015; Gómez, Galeano y Jaramillo, 2015). En este sentido se procedió a delimitar el tema, acumular la información, organizar la data y elaborar un esquema conceptual para finalmente completar el análisis de lo encontrado.

Habiendo delimitado el tema como el concepto de alfabetización dentro del sistema educativo venezolano se comenzó con un estudio de documentos oficiales como el Currículo Básico Nacional de 1997, el Currículo del Subsistema de Educación Primaria del año 2007, las Orientaciones Pedagógicas del Ministerio del Poder Popular para la Educación (MPPE) 2017-2018 y la Memoria y cuenta del Ministerio del Poder Popular para la Educación 2013, y otros que fueron contrastados con autores versados en el tema, entre los que se pueden mencionar a Ortiz y Fleires (2006), Ortiz (2013), Cámara Venezolana de la Educación Privada (2014) y Uzcátegui (2017) por nombrar algunos. 
A partir de esta recolección, y de elaborar el esquema conceptual de la data (Tabla 1) se derivó un análisis en el que se pudo evidenciar varios aspectos. En primer lugar, estuvo claro que no existe en las fuentes de información oficiales un concepto actual y unificado sobre la alfabetización fuera de la descripción metodológica expuesta en los currículos oficiales y los documentos del proyecto de Escuelas Bolivarianas (Ministerio del Poder Popular para la Educación, 2004 y 2007).

\begin{tabular}{|l|l|l|l|l|}
\cline { 2 - 5 } \multicolumn{1}{c|}{} & La Escuela Nueva & Asociacionismo & Constructivismo & \multicolumn{1}{c|}{$\begin{array}{c}\text { Escuelas } \\
\text { Bolivarianas }\end{array}$} \\
\hline Período & \multicolumn{1}{c|}{$(1936-1969)$} & $(1963-1990)$ & $(1990-2000)$ & (2000-Presente) \\
\hline Método & $\begin{array}{l}\text {-Método global o } \\
\text { ideovisual } \\
\text { _Unidades de } \\
\text { lectura }\end{array}$ & $\begin{array}{l}\text {-Modelo de } \\
\text { destrezas }\end{array}$ & Constructivismo & $\begin{array}{l}\text { EEnseñanza por } \\
\text { áreas- } \\
\text { Constructivismo }\end{array}$ \\
\hline
\end{tabular}

Tabla 1. Esquema conceptual de la Alfabetización. (Fuente: Elaboración propia)

En segundo lugar, se destaca que la carencia de un concepto oficial de alfabetización emanado del Ministerio del Poder Popular para la Educación trae como consecuencia que tampoco exista un método adaptado a las necesidades particulares de la población venezolana, por lo que queda a cargo de cada docente en particular la decisión del método más apropiado a aplicar, lo que puede resultar en inconsistencias en el desarrollo de la lectura y escritura entre la población estudiantil, incluso dentro de una misma institución (Arévalo, Lugo y Pineda, 2016).

Así mismo, fue evidente en esta revisión que las últimas reformas curriculares estuvieron más orientadas a reformar la estructura general de las asignaturas cursadas en la educación primaria y secundaria, la configuración de la comunidad educativa y las temáticas a abordar más no el método de enseñanza a ningún nivel (Ministerio del Poder Popular para la Educación, 2017).

Finalmente, a partir de esta revisión se pudo elaborar una ruta histórica de la alfabetización en el Sistema Educativo Venezolano, lo que sirvió para ilustrar más 
detalladamente la evolución del concepto desde el punto de vista de las políticas del Estado.

\section{Resultados y discusión}

\section{La alfabetización inicial en Venezuela}

La evolución del concepto de alfabetización inicial transita la ruta de la transformación del Sistema Educativo Venezolano. Es propicio así, iniciar el recorrido de la alfabetización y en el año de 1870, cuando según cifras de la Cámara Venezolana de la Educación Privada (CAVEP, 2014) se atendían aproximadamente 3700 niños en alrededor de 100 escuelas. En el mismo año, durante la presidencia de Antonio Guzmán Blanco, se decreta la instrucción obligatoria y la creación de la Dirección de Instrucción Pública, se fortalece el alcance de la educación con la creación de 336 escuelas federales y 383 escuelas municipales.

El mencionado decreto comprende la enseñanza en los valores de moralidad, lectura y escritura del idioma oficial de la nación, matemática básica, sistema métrico y las leyes expuestas en la constitución. De este modo inicia el crecimiento del Sistema Educativo Venezolano, que alcanzaría su punto de transformación a partir de 1950 con la llegada del período democrático.

Falcón de Ovalles (2003) divide la mencionada historia en tres etapas:

La Escuela Nueva (1936-1969)

El Asociacionismo (1969-1990)

El Constructivismo (1990-2000)

Esta división llama a la actualización tomando en consideración la transformación sociopolítica ocurrida en Venezuela a partir del año 2000, de la que evidentemente no escapó el sistema educativo. Por tanto, a la anterior numeración se añade una cuarta etapa:

Las Escuelas Bolivarianas (2000-)

La Escuela Nueva (1936-1969):

Este período inicia posterior a la muerte de Juan Vicente Gómez y culmina en 1969 bien entrado el período democrático. A pesar de que durante el último tercio del siglo XIX Guzmán Blanco había decretado la obligatoriedad de la educación básica, para el final de 
la dictadura gomecista el sistema educativo se encontraba en franco deterioro. Uno de los principales problemas de la educación venezolana para este período era sin duda la carencia de docentes apropiadamente formados para la profesión. A esto se le suma que solamente 1 de cada 5 niños en edad escolar estaba inscrito en la escuela primaria y el presupuesto educativo de la nación no superaba el 4 \% (CAVEP, 2014).

En este orden de ideas, el modelo de Escuela Nueva tuvo su origen en el pensamiento de Dewey, quien pone al niño en el centro de interés pedagógico reconociéndolo como sujeto activo de su enseñanza y dándole un papel protagónico (Vélez, 2017). La Escuela Nueva se presenta como una comunidad en miniatura en la que el niño aprende de la experiencia (Ortiz, 2013).

En la Escuela Nueva, los dictados, las copias y el silabeo son sometidos al escrutinio de los principios generales del Método Global o Ideovisual, enunciado por Ovidio Decroly, y las Unidades de Lectura. La idea era enseñar la lengua escrita a partir de la motivación, dejando de lado el tedioso esfuerzo de internalizar la decodificación de cada palabra del alfabeto. Se busca así facilitar el proceso a través de estrategias como el uso de ilustraciones y el juego que harán que el estudiante avance a su ritmo y autónomamente a la comprensión (Xu y Brown, 2016).

El Método Global o Ideovisual, aplicado durante esta etapa, comulgaba con la flexibilidad y el paidocentrismo y no dictaminaba un concepto o metodología específica para la alfabetización pues esta debía adaptarse a cada grupo de estudiantes y a sus características específicas (De Mello y Porta, 2017).

La aplicación de las unidades de lectura también contribuyó a lograr cierta unidad en el tratamiento de la enseñanza de la lectura y a superar el caos en el que se debatían los docentes sin suficiente preparación académica y científica. No obstante, se le abrió la puerta al asociacionismo, que a través del análisis fónico y los ejercicios repetitivos de decodificación terminó por establecer el modelo de destrezas dando paso a la segunda fase de transformación de la alfabetización en el SEV.

El Asociacionismo (1969-1990)

En esta etapa de la alfabetización del Sistema Educativo Venezolano comienza con el ascenso de las tendencias positivistas en la educación las cuales argumentaban una 
concepción tecnicista en los procesos de enseñanza aprendizaje dándole un tratamiento científico a todos sus componentes traduciéndolos en elementos objetivos y mesurables.

Con esto en mente, la alfabetización es vista como un instrumento, que si bien guarda un relevante ingrediente sociocultural, es en definitiva una destreza que es aprendida y perfeccionada a través de la observación y la imitación, la repetición y el refuerzo cotidiano (Porta e Isón, 2011). Venezuela, entonces, no escapó de la inclinación mundial hacia metodologías de aprendizaje que redujeran el espacio a lo abstracto, en las que se especificara detalladamente lo que se esperaba de los alumnos y, por consiguiente, lo que el docente debía hacer para provocar la respuesta deseada en su grupo. Es así como esta época se caracterizó por planificaciones minuciosas y escrupulosas listas de objetivos (Villarroel, 2015).

En la etapa del asociacionismo, la alfabetización era concebida como una destreza compleja compuesta por subdestrezas (decodificación y comprensión) por lo que para su manejo a nivel diestro se necesitaba ir conquistando secuencialmente cada subdestreza hasta llegar al conocimiento del todo (Many, 2009). Según el modelo de destrezas se comenzaba con la subdestreza de decodificación tomando en primer lugar el manejo de las unidades mínimas del lenguaje; reforzando cada logro y conducta deseable hasta llegar gradual y sistemáticamente a la subdestreza de comprensión.

En este orden de ideas, una de las grandes diferencias entre el Método Global y el Modelo de Destrezas era la claridad de los procedimientos de enseñanza de este último que, si bien no dictaminaba una receta puntualizada, sí pintaba un panorama más configurado para la alfabetización. Es así como en el año 1990, el SEV ingresa en la siguiente etapa de su transformación buscando dejar definitivamente de lado las prácticas mecanicistas y el exceso de objetividad que aspiraba a cuadricular la fluida naturaleza humana.

\section{El Constructivismo (1990-2000)}

Fueron muchos los factores científicos, sociales y políticos que propiciaron el descenso de los métodos conductistas y asociacionistas. Desde los esfuerzos de académicos, como los de los fundadores de la Universidad Pedagógica Experimental Libertador (UPEL) en 1983, entre otras, hasta legislaciones del Ministerio de Educación, pasando por las indetenibles dinámicas socioculturales del país, de cierto modo 
confabularon para abrir la puerta al constructivismo como paradigma de enseñanza y aprendizaje.

Hasta 1990 la alfabetización era concebida como una asociación entre estímulos (letras) y respuestas (sonidos) pero con la llegada del constructivismo la enseñanza inicial de la lectura y la escritura se empieza a abordar como una construcción cuyo paso va dictado por el estadio de desarrollo del estudiante y su realidad (Padrón, 2013 y Vargas, 2016). Esta realidad toma la forma de esquemas (Conocimientos previos) y su interacción con el nivel de desarrollo desencadena procesos de asimilación y acomodación en los que también pesa la influencia de la socialización (Figueroa, Muñoz, Lozano y Zavala, 2016).

Con este basamento, en el año 1997 se reforma el currículo educativo venezolano abandonando los axiomas asociacionistas y conductistas para abrirse hacia el constructivismo. El nuevo Currículo Básico Nacional (CBN) para la primera y segunda etapa de educación pretendía desarrollar la competencia comunicativa de los estudiantes de manera funcional, respaldando la premisa de que la alfabetización se lograba participando en actos de lectura y escritura "que lleven a la construcción de significados y a la comprensión global en el marco de situaciones significativas y de uso real" (Ministerio de Educación, 1997 citado en Ortiz y Fleires, 2006, p. 45).

En el enfoque constructivista lo cognitivo y lo lingüístico deben entrelazarse en la enseñanza y aprendizaje del texto escrito (Colliander, Ahn y Anderson, 2018). La reforma curricular de 1997 propuso una metodología general para la alfabetización, buscado motivar la comprensión y producción significativa de textos, así como la formación de escritores y lectores expertos que especificaron desde el inicio de cada evento o ejercicio su propósito comunicativo (Padrón, 2014).

La aplicación del CBN y la Reforma Curricular tuvieron corto tiempo de vida en el SEV, pues el 6 de diciembre de 1998 con la victoria electoral de Hugo Chávez Frías, Venezuela cerró un ciclo en su historia y abrió otro que traería grandes cambios y transformaciones en todos los sectores sociales, políticos y económicos del país (Uzcátegui, 2017).

Particularmente en el sector educativo, a partir del año 1999 se pone en marcha la Constituyente Educativa relanzando los proyectos de jornada escolar completa (8 am$3 \mathrm{pm})$, y específicamente el 15 de septiembre de ese año se promulga el decreto de creación 
de las Escuelas Bolivarianas (EB) que vendrán a representar un cambio de concepciones ideológicas y prácticas del SEV.

Las Escuelas Bolivarianas (2000-)

Las décadas de 1980-1990 en América Latina fueron de grandes cambios, a los cuales no escapó Venezuela. Rodríguez (2008) relata que ya para el año 1990 tanto el ejecutivo nacional como los gobiernos estatales habían empezado a implementar medidas para atender a la población nacional en riesgo y garantizar su prosecución escolar. En este orden de ideas, en los estados Bolívar, Mérida y Aragua se implementaron proyectos educativos destinados al mejoramiento tanto de las necesidades alimenticias de la población como de la calidad educativa. El Plan Especial de Educación del Estado Bolívar (1989).Las Escuelas Integrales del estado Mérida y Las Escuelas Solidarias del Estado Aragua (1992), funcionaban en su mayoría a jornada completa, avocándose a la aplicación de metodologías que integraran al estudiante y la comunidad en el proceso de aprendizaje al tiempo que, proveyendo los recursos alimenticios básicos de los estudiantes, se garantizara su egreso formal de la educación básica (Uzcátegui, 2017).

Señala Rodríguez (2008) a estos programas como los antecedentes de la Educación Bolivariana, la cual fue concebida como:

Un proceso social que se crea en colectivo como expresión de cultura y su finalidad es fomentar el pensamiento liberador, creador y transformador, así como la reflexión crítica la participación ciudadana y los sentimientos de honor, probidad, amor a la patria, a las leyes y al trabajo todo ello para lograr el desarrollo endógeno de los pueblos (Ministerio del Poder Popular para la Educación, 2007, pp 9-11).

El Sistema Educativo Bolivariano (SEB) se comienza entonces a aplicar, a partir del 15 de noviembre de 1999 caracterizándose por: atender a los estudiantes en jornada completa de 8 horas, integrar a la escuela-comunidad y una renovación pedagógica permanente. Comparativamente, las Escuelas Bolivarianas rescatan varias características de 
filosofías ya superadas como las de la Escuela Nueva, sobre todo en lo que se refiere a la enseñanza por unidades de lectura que en esta nueva etapa pasó a llamarse enseñanza por áreas. El SEB no privilegia ningún método de alfabetización sobre otro. De hecho, no existe dentro de sus principios un concepto de alfabetización, pues el método idóneo será aquel que se adapte mejor al grupo de estudiantes y su contexto social (Abreu, 2018).

En este sentido, el desarrollo de la competencia lectora se hace progresivamente durante toda la instrucción primaria añadiendo nuevas destrezas a medida que avance el desarrollo psicomotor de los estudiantes, esto en base a las finalidades de cada etapa, tomando en cuenta que la lectura debe ante todo promover el desarrollo de la ciudadanía activa y crítica orientada a los valores base de la educación bolivariana (participación ciudadana, honor, probidad, amor a la patria, a las leyes y al trabajo) (Ministerio del Poder Popular para la Educación, 2017).

Con este propósito se regula el uso de la Colección Bicentenario-Cardenalito, la cual se concibe como guía de lectura para 1ro a 6to grado. Se debe hacer énfasis en el hecho de que el libro Cardenalito se concibe como una guía de lectura, porque según las finalidades del SEB para el Área de Lenguaje, Comunicación y Cultura lo más importante es que los niños estén siempre en contacto con textos auténticos que promuevan la comunicación real (Ministerio del Poder Popular para la Educación, 2004).

Finalmente, la alfabetización en Venezuela se ha ido nutriendo a través de los años de distintas corrientes filosóficas tanto nacionales como extranjeras que responden al momento histórico y político de la nación, lo que le ha brindado distintos enfoques durante su ejecución; que en su momento han tenido una mayor influencia que otro.

\section{Conclusiones}

A través de los años el concepto de alfabetización en Venezuela, ha estado alejado del verdadero carácter comunicativo de la práctica de la lengua escrita, orientándose más hacia el desarrollo de destrezas decodificadoras. Esto ha traído como consecuencia que los individuos perciban la escritura o la lectura como una actividad tediosa y ajena a su cotidianidad. Algo que se lleva a cabo para cumplir con algún requisito o asignación y no como un medio para expresarse y conectarse con su entorno. 
Esta exploración de los principales aspectos teóricos y epistemológicos relacionados con la alfabetización deja evidencia de que en efecto no existe un concepto definido de alfabetización dentro del Sistema Educativo Venezolano, lo que implica que no existe un método definido que guíe a los docentes en sus prácticas alfabetizadoras más allá de las recomendaciones teóricas o ideológicas emanadas de los entes oficiales. La presencia de una clara conceptualización es de vital importancia pues, como se ha indicado la presencia de un concepto claro marcará y moldeará políticas, presupuesto y todas las acciones educativas de un país.

Se concluye a partir de este estudio que, la alfabetización inicial en Venezuela ha sufrido cambios metodológicos y teóricos a lo largo de su historia. Estos cambios han ido de la mano con los cambios en la política y economía de la nación ligando las prácticas educativas a la ideología del Estado lo que sin duda da forma a unas prácticas alfabetizadoras que no necesariamente abrazan el componente comunicativo del lenguaje escrito, sino que persiguen objetivos políticos emanados desde el gobierno central.

Con esto en mente, se recomienda usar las nociones aquí planteadas como hoja de ruta inicial para una futura indagación más profunda que pueda iniciar una transformación de las mencionadas conductas hacia otras en las que se aprecie en su totalidad todo lo que los seres humanos pueden lograr a través de la lengua escrita, bajo la premisa del cuestionamiento de la realidad, la curiosidad y la valoración del lenguaje escrito como herramienta de crecimiento personal y profesional.

\section{Referencias Bibliográficas}

Abreu, A. (2018). "Una mirada al pensamiento educativo de Luis Beltrán Prieto Figueroa”. Educare. Vol. 22, $\mathrm{N}^{\circ} 3$, pp. 140-154.

Arévalo, J., Lugo, M. y Pineda, C. (2016). “Diagnóstico para el proyecto La escuela y la lengua escrita" Proyecto de Investigación avalado por el Decanato de Investigación UNEFM. Coro, Venezuela: Universidad Nacional Experimental Francisco de Miranda.

Bernal, D., Martínez, L., Parra, A. y Jiménez, L. (2015). “Investigación documental sobre calidad de la educación en instituciones educativas del contexto Iberoamericano". Revista Entramados-Educación y Sociedad. Año 2, º 2, pp. 107-124. 
Braslavsky, B. (2003). “¿Qué se entiende por alfabetización?”. Lectura y Vida. Año: 24, N 2, pp. 1-17.

Cassany, D. (2015). "Las ortografías en internet: exploración datos y reflexiones”, en Montoro, E. Estudios sobre ortografia del Español. Lugo: Axax, pp. 13-26.

CAVEP (2014). La transformación del sistema educativo venezolano. Un reto ineludible. Caracas: CAVEP.

Colliander, H., Ahn, S. \& Andersson, P. (2018). "Actions and conceptions: Exploring initial literacy practices for adults". Journal of language, identity and education. Vol. 17, N5, pp. 306-319.

De Mello, R. y Porta, M. (2017). “Estrategias pedagógicas de alfabetización y su efecto en el aprendizaje inicial de la lecto-escritura". Revista de orientación educacional. Vol. $31, \mathrm{~N}^{\circ} 60$, pp. $22-45$.

Falcón de Ovalles, J. (2003). La enseñanza de la lectura en Venezuela: De la escuela nueva al constructivismo y el análisis del texto. Caracas: Ediciones Vicerrectorado de Investigación y Postgrado UPEL.

Ferreiro, E. (2016). Pasado y presente de los verbos leer y escribir. México: Fondo de Cultura Económica.

Figueroa, H., Muñoz, K., Lozano, E. y Zavala, D. (2016). “Análisis crítico del conductismo y constructivismo, como teorías de aprendizaje en educación”. Revista Órbita Pedagógica. Vol. 4, N¹, pp. 1-11.

Gómez, M., Galeano, C. y Jaramillo, D. (2015). “El estado del arte: Una metodología de investigación”. Revista Colombiana de Ciencias Sociales. Vol. 6, N², pp. 423-442.

Hernándo, A y García, I. (2016). “Identidad y escritura en redes sociales”. Cervantes. Vol. $15, \mathrm{~N}^{\circ} 1$, pp. 461-474.

Many, J. (2009). The handbook of instructional practices for literacy teachers-educators. Examples and reflections from the teaching lives of literacy scholars. New Jersey: Routledge, Taylor-Francis Library. 
Ministerio de Educación (1997). Currículo Básico Nacional. Caracas.

Ministerio del Poder Popular para la Educación (2004). La educación bolivariana. Política, programas y acciones. Cumpliendo las metas del milenio. Caracas.

Ministerio del Poder Popular para la Educación (2007). Currículo del Subsistema de Educación Primaria Bolivariana. Caracas.

Ministerio del Poder Popular para la Educación (2013). Ministerio del Poder Popular para la Educación. Memoria y cuenta. Caracas.

Ministerio del Poder Popular para la Educación (2017). Orientaciones pedagógicas año escolar 2017-2018. Caracas.

Ortiz, A. (2013). "Modelos pedagógicos y teorías de aprendizaje". Ediciones de la U. Colombia: Universidad de Magdalena.

Ortiz, M. y Fleires, L. (2006). "Principios didácticos para la enseñanza de la lectura durante la alfabetización inicial". Revista de Artes y Humanidades UNICA. Año 8, $\mathrm{N}^{\circ} 18$, pp. 42-59.

Owens, R. (2016). Language development: An introduction. London: Pearson.

Padrón, J. (2013). ¿Alfabetizaciones múltiples o alfabetización inicial? Caracas: Universidad Nacional Abierta.

Padrón, J. (2014). Alfabetización integral. La UNA como escenario. Caracas: Vicerrectorado académico de la UNA.

Porta, M. e Isón, M. (2011). "Hacia un enfoque comprensivo del aprendizaje lingüístico inicial como proceso cognitivo". Revista Iberoamericana de educación. Año: 2011, № 5, pp. 243-260.

Portolés, J. (2016). La censura de la palabra. Estudio de pragmática y análisis del discurso. Valencia, España: PUV. Universitat de Valencia.

Rodríguez, N. (2008). "Trayectoria del proyecto de Escuelas Bolivarianas”. EducereInvestigación Arbitrada. Año 12, $\mathrm{N}^{\circ} 42$, pp. 563-574. 
UNESCO (2006). La alfabetización un factor de vida: Educación para todos. El informe de seguimiento. París: UNESCO.

Uzcátegui, R. (2017). Cronología de la Educación en Venezuela. Siglo XVI-2016. Para el magisterio venezolano. Caracas: EE-CIES-FHE-UCV.

Vargas, A. (2016). "Redes sociales, literacidad e identidad (es): El caso de Facebook". Revista Colombiana de Lingüística Aplicada. Vol. 18, №1, pp. 11-24.

Vélez, I. (2017). "Políticas públicas en alfabetización mediática e informacional: El Legado de John Dewey”. Revista Fuentes. Vol. 19, N², pp. 39-57.

Villarroel, P. (2015). "Recorrido metodológico en educación inicial”. Sophia. Vol. 192015, pp. 154-170.

$\mathrm{Xu}$, Y. \& Brown, G. (2016). "Teacher assessment literacy in practice. A reconceptualization”. Teaching and Teacher Education. Vol. 58-2016, pp. 149-162. 